\title{
Genomic insights into divergence and dual domestication of cultivated allotetraploid cottons
}

\author{
Lei Fang ${ }^{1 \dagger}$, Hao Gong ${ }^{2 \dagger}$, Yan Hu ${ }^{1 \dagger}$, Chunxiao Liu ${ }^{1 \dagger}$, Baoliang Zhou', Tao Huang ${ }^{2}$, Yangkun Wang ${ }^{1}$, Shuqi Chen ${ }^{1}$, \\ David D. Fang ${ }^{3}$, Xiongming Dư ${ }^{4}$, Hong Chen ${ }^{5}$, Jiedan Chen', Sen Wang ${ }^{1}$, Qiong Wang ${ }^{1}$, Qun Wan ${ }^{1}$, Bingliang Liu', \\ Mengqiao Pan ${ }^{1}$, Lijing Chang ${ }^{1}$, Huaitong Wu' ${ }^{1}$, Gaofu Mei ${ }^{1}$, Dan Xiang ${ }^{1}$, Xinghe Li $^{1}$, Caiping Cai ${ }^{1}$, Xiefei Zhu' ${ }^{1}$, \\ Z. Jeffrey Chen ${ }^{1,6}$, Bin Han ${ }^{2}$, Xiaoya Chen ${ }^{7}$, Wangzhen Guo ${ }^{1}$, Tianzhen Zhang ${ }^{1,8^{*}}$ and Xuehui Huang ${ }^{2,9^{*}}$
}

\begin{abstract}
Background: Cotton has been cultivated and used to make fabrics for at least 7000 years. Two allotetraploid species of great commercial importance, Gossypium hirsutum and Gossypium barbadense, were domesticated after polyploidization and are cultivated worldwide. Although the overall genetic diversity between these two cultivated species has been studied with limited accessions, their population structure and genetic variations remain largely unknown.

Results: We resequence the genomes of 147 cotton accessions, including diverse wild relatives, landraces, and modern cultivars, and construct a comprehensive variation map to provide genomic insights into the divergence and dual domestication of these two important cultivated tetraploid cotton species. Phylogenetic analysis shows two divergent groups for $G$. hirsutum and G. barbadense, suggesting a dual domestication processes in tetraploid cottons. In spite of the strong genetic divergence, a small number of interspecific reciprocal introgression events are found between these species and the introgression pattern is significantly biased towards the gene flow from G. hirsutum into G. barbadense. We identify selective sweeps, some of which are associated with relatively highly expressed genes for fiber development and seed germination.
\end{abstract}

Conclusions: We report a comprehensive analysis of the evolution and domestication history of allotetraploid cottons based on the whole genomic variation between G. hirsutum and G. barbadense and between wild accessions and modern cultivars. These results provide genomic bases for improving cotton production and for further evolution analysis of polyploid crops.

Keywords: Allotetraploid cottons, Resequencing, Divergence, Domestication

\section{Background}

Cotton (Gossypium spp.) is the most important natural fiber and edible oil crop in the world. The genus Gossypium includes around 45 diploid $(2 \mathrm{n}=2 \mathrm{x}=26)$ and five allotetraploid $(2 \mathrm{n}=4 \mathrm{x}=52)$ species. The allotetraploids that were present 1-1.5 million years ago (MYA)

\footnotetext{
* Correspondence: cotton@njau.edu.cn; xhhuang@shnu.edu.cn

${ }^{\dagger}$ Equal contributors

'State Key Laboratory of Crop Genetics and Germplasm Enhancement, Cotton Hybrid R \& D Engineering Center (the Ministry of Education), Nanjing Agricultural University, Nanjing 210095, China

${ }^{2}$ National Center for Gene Research, Institute of Plant Physiology and Ecology, Shanghai Institutes for Biological Sciences, Chinese Academy of Sciences, Shanghai 200233, China

Full list of author information is available at the end of the article
}

originated from one hybridization event between an extant progenitor of Gossypium herbaceum $\left(\mathrm{A}_{1}\right)$ or Gossypium arboreum $\left(\mathrm{A}_{2}\right)$ and another progenitor, Gossypium raimondii Ulbrich $\left(\mathrm{D}_{5}\right)$ [1-3]. Gossypium wild relatives grew primarily as perennial upright shrubs or small trees and existed in various stages of domestication as feral derivatives that had established self-perpetuating populations in human-modified environments such as road sides, field edges, and dooryards [4]. Cotton is a unique example of crop domestication that occurred in two Old World diploids, G. herbaceum L. and G. arboreum L. and two New World allotetraploids, Gossypium hirsutum and Gossypium barbadense, in four different pre-historical cultures [4]. Under long-term human 
selection of a wide range of morphological and physiological traits, the two tetraploid species, G. hirsutum and G. barbadense, have been domesticated and cultivated. However, photoperiod sensitivity in long-lived perennial species with a slow rate of plant development and seed emergence and the broad spectrum of fruiting habits in cultivars have been under investigated [5-7].

Modern G. hirsutum cultivars (Upland cotton) have high-yield properties and dominate more than $90 \%$ of worldwide cotton production, while G. barbadense, characterized by its extra-long staple (ELS) and strong and fine fibers accounts for less than 10\% [8]. G. hirsutum is native to the Mesoamerican and the Caribbean regions, and G. barbadense is indigenous to the coastal areas of Peru $[9,10]$. Through intensive study of germplasm collections, Hutchinson [11] identified one wild and six domesticated (not botanical varieties) races of G. hirsutum based mainly on their morphologies and distinct geographic distributions. Modern Upland cotton has been further improved in the southern United States from domesticated early-cropping perennials through extensive human selection to produce a common set of agronomic features known as "domestication syndrome" traits [12]. These traits include an annual growth habit and photoperiod insensitivity [5], decreased seed dormancy [6], a large boll size and number per plant [1], and superior fiber quality [13]. The genetic diversity of allotetraploid cottons has been studied for decades using pedigree information and morphologies [14, 15], biochemical markers [7, 16], and DNA-based markers [17-20]. Genomic insights into variation within and between allotetraploid cotton species are limited by the lack of known allotetraploid genome sequences. To resolve this, we resequenced and conducted genomic analysis of 147 cotton accessions with different origins after sequencing the genome of the genetic standard Upland cotton line, TM-1 [21]. Until now, only a few candidate genes related to cotton lint yield and fiber quality have been functionally characterized. So, we integrated the expression profiling data, quantitative trait loci (QTL) mapping, and function annotations with orthologs in Arabidopsis to conduct rapid identification of genes associated with domestication, especially fiber development and seed germination. The present research provides genome-wide level insights into genetic divergence and dual domestication of cultivated tetraploid cottons.

\section{Results and discussion}

\section{Genetic diversity}

Upland and Sea Island varieties were established in the seaboard colonies of the southeastern United States by the mid-18th century and the Egyptian cottons in the Nile Delta by the early 19th century. So, we sampled 147 G. hirsutum and G. barbadense accessions, including wild species, races, landraces, and modern improved cultivars, from different geographic locations, representing the long history of cotton domestication and breeding throughout the world (Table 1; Additional file 1: Figure S1; Additional file 2: Table S1). Close relatives of the allotetraploid cotton species, Gossypium tomentosum $(\mathrm{AD})_{3}$, Gossypium mustelinum (AD) $)_{4}$, and Gossypium darwinii $(\mathrm{AD})_{5}$, as well as Thespesia populneoides (Roxb.) Kostelas, which is closely related to the genus Gossypium in the Malvaceae family, were all included as outgroups. We resequenced all 147 accessions with approximately fivefold coverage, generating a total of 1.8 terabases of raw sequence data, and aligned the reads to the reference genome sequence of TM-1 [21] to identify sequence variants (Table 1). We used direct genome sequence comparison and PCR-based sequencing strategies to validate the quality of the called single nucleotide polymorphisms (SNPs). Two recently sequenced accessions of G. barbadense cv. Xinhai 21 (XH21) [22] and G. hirsutum acc.TM-1 [21] in our sequence panel were used as controls. We checked the called SNPs from our sequence panel against two assembled genome sequences and found the accuracy of SNP calling to be $96.2 \%$ for XH21 and $99.1 \%$ for TM-1, with a low missing data rate (6.8\%). We further randomly selected 68 SNPs to carry out PCR-based sequencing in 11 accessions, each randomly selected from one cluster of the phylogenetic tree constructed with 147 accessions, and found that the accuracy was $95.0 \%$ (Additional file 1:

Table 1 Summary of sequencing of and variations in G. hirsutum and G. barbadense

\begin{tabular}{lllcll}
\hline Group & Accessions (number) & Raw data $(\mathrm{Gb})$ & Raw data depth & $\begin{array}{l}\text { Uniquely mapping rate } \\
\text { to the A subgenome }\end{array}$ & $\begin{array}{l}\text { Uniquely mapping rate } \\
\text { to the D subgenome }\end{array}$ \\
\hline G. hirsutum cultivars & 52 & 734 & 5.64 & $36.1 \%$ & $24.9 \%$ \\
G. hirsutum races & 33 & 442 & 5.35 & $34.3 \%$ & $22.4 \%$ \\
G. barbadense cultivars & 52 & 534 & 4.10 & $36.6 \%$ & $24.3 \%$ \\
Others $^{\text {a }}$ & 10 & 132 & 5.19 & $35.2 \%$ & $21.0 \%$ \\
Total & 147 & 1,842 & 5.17 & $36.2 \%$ & $23.9 \%$ \\
\hline
\end{tabular}

${ }^{a}$ Others includes four G. barbadense races, Kaiyuanlihemumian, Yuanmoulihemumian, Alabolihemumian, and Kaiyuanlianhemumian, and close relatives Thespesia populneoides (Roxb.) Kostel, Gossypium purpurascens, G. mustelinum, G. darwinii, and an Indian cultivar, NV50-70 
Figure S2; Additional file 3: Table S2; Additional file 4: Table S3). Therefore, the quality should be reliable enough for follow-up phylogenetic and population genetic analyses.

Of the sequenced reads, 36.2 and $23.9 \%$ were uniquely mapped to the A and D subgenomes of the TM-1 reference genome (1.9-Gb oriented scaffold), respectively (Table 1). Additionally, $10.5 \%$ of the total reads were mapped to the A subgenome unoriented scaffolds and $1.9 \%$ of the total reads were mapped to the D subgenome unoriented scaffolds; we did not use these A or D subgenome unoriented scaffolds for further analysis. Moreover, $23.4 \%$ of the total reads were mapped to no or multiple locations, which may be caused by the high proportion of repeated sequence $(67.2 \%)$ or the highly homoeologous regions between the $\mathrm{A}$ and $\mathrm{D}$ subgenome in cotton. Only $4.1 \%$ of the total reads were mapped to the unclassified scaffolds, which had little effect on our analysis.

Overall, we identified 16,377,749 non-unique SNPs, defined as those with the variant occurring in at least two accessions and 144,662 non-unique indels (1 bp-8 kb; Additional file 5: Table S4). Of these indels, 16,879 with $>50$-bp indels were identified as structural variants (SVs; Additional file 1: Figure S3; Additional file 6: Table S5). For instance, the SV (2992 bp) identified in chromosome D09 from 44,118,172 to 44,121,164 bp could be detected in 37 accessions. These variants were distributed across all 26 chromosomes, with an average density of 8.5 SNPs per kilobase (Additional file 7: Table S6). The SNP density in the A subgenome (9.2 SNPs per kilobase) was higher than that in the D subgenome (7.4 SNPs per kilobase). By analyzing the allele frequency of each SNP site in the 147 accessions, we identified 7,993,856 common SNPs, each with an allele frequency of $>5 \%$, including 3,203,112 intraspecific SNPs in G. hirsutum, 3,770,221 in G. barbadense, and 2,752,128 ( 34.4\%) nearly fixed interspecific SNPs (SNPs with an allele frequency of $>95 \%$ in G. hirsutum or G. barbadense and $<5 \%$ in the other species (Additional file 1: Figure S4).

\section{Dual domestication of cultivated allotetraploid cottons}

The whole-genome SNP data were used to investigate the phylogenetic relationships between all allotetraploid cotton collections (Fig. 1a; Additional file 8: Dataset 1). The subsequently produced neighbor-joining (NJ) tree resulted in two largely divergent clades: the G. hirsutum clade $(n=85)$ and the $G$. barbadense clade $(n=52)$, consistent with a previous study, although with a limited number of accessions [23]. Both studies suggested a strong divergence between G. hirsutum and G. barbadense. Model-based analyses of population structure using STRUCTURE revealed that there were two different components between G. barbadense and G. hirsutum when $\mathrm{K}$ (the number of populations modeled) was set to 2. However, when $\mathrm{K}$ was set to 3 , there were three different components: G. barbadense cultivars, G. hirsutum cultivars, and G. hirsutum races (Fig. 1b). This model-based result, along with that from principal component analysis (Fig. 1c), agreed well with the pattern in the phylogenetic tree. The outgroup type comprised ten accessions in total, including T. populneoides, G. tomentosum (Hawaiian Islands), G. darwinii (Galapagos Islands), and seven tetraploid accessions that might have resulted from genetic introgressions from wild progenitors or from historical interspecific crossing between G. hirsutum and G. barbadense (Additional file 2: Table S1). No clear separation existed between the seven races (33 accessions in total) in G. hirsutum, which was likely due to human-mediated accession expansion, bringing formerly isolated races into mixed and overlapping distributions (Fig. 1d). However, one punctatum race from Egypt and one latifolium race from Chiapas were most closely related to G. hirsutum cultivars (Fig. 1; Additional file 8: Dataset 1). Some African and Indian cultivars were classified into one landrace subgroup, which was closely related to the true annual forms of punctatum grown in Africa, further supporting the early cropping of race punctatum in the Old World [11]. Punctatum is a race originally found inland on the Yucatan peninsula. Whether annual forms of punctatum were developed before or after its introduction into Africa remains to be explored. These genomic data revealed at least two origins of upland cotton in the Old World and the New World; punctatum in America or Africa and latifolium in America, consistent with the domestication and improvement history of upland cotton $[1,2,11,18]$.

The origins of modern cultivated G. barbadense are complex and somewhat obscure. Unlike G. hirsutum, which exists in both wild and cultivated states, G. barbadense is found only in cultivars. The present research provides genomic evidence that $G$. barbadense is indigenous to Peru and Brazil since those primitive landraces of $G$. barbadense native to Brazil and Peru together with West of the Andes and Sea Island cotton were classified into one subgroup (Fig. 1e). It suggests a probable center of origin in northwestern South America, consistent with archeological records [24]. All modern ELS cultivars were classified into three subgroups: Egyptian, American Pima, and Central Asia cottons.

\section{Genomic divergence between G. hirsutum and G. barbadense}

Much of the genetic diversity of cotton can be quantified by the frequency of SNPs. In addition to 322,285 codingregion SNPs (cSNPs) and 173,334 intronic-region SNPs involved in 56,401 predicted genes [21], the majority (93.8\%) of the 7,993,856 common SNPs were located in intergenic regions (Additional file 1: Figure S5). The allele 
a

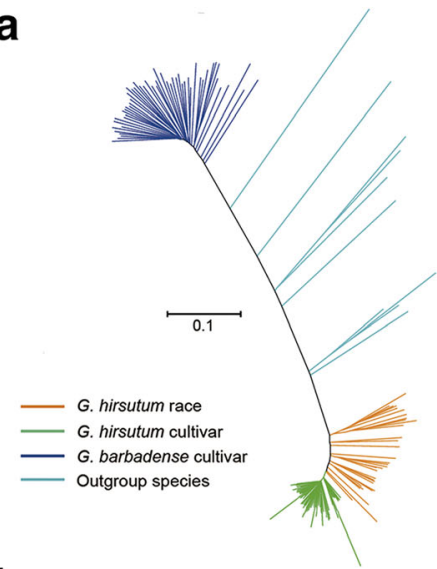

b

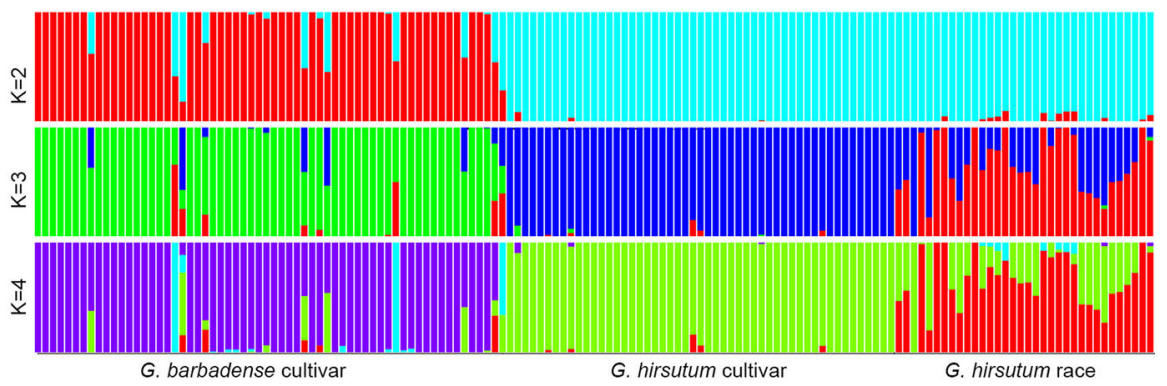

d

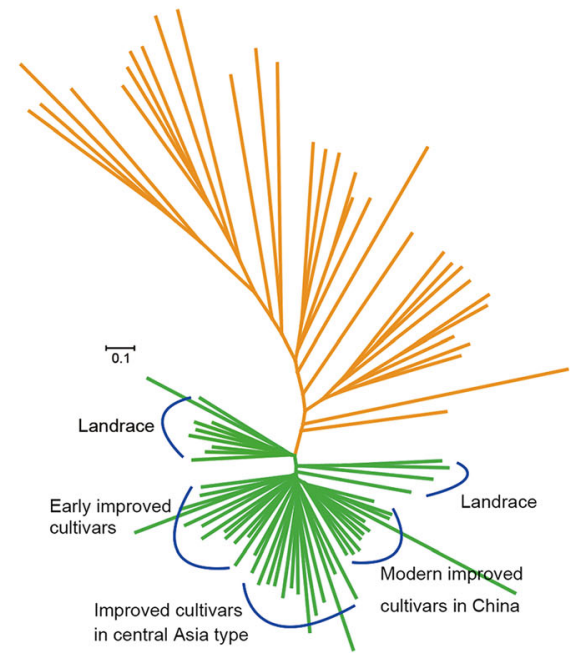

C

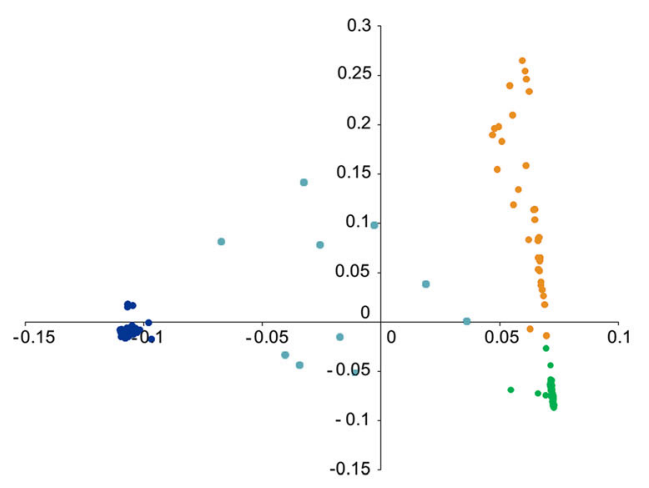

e

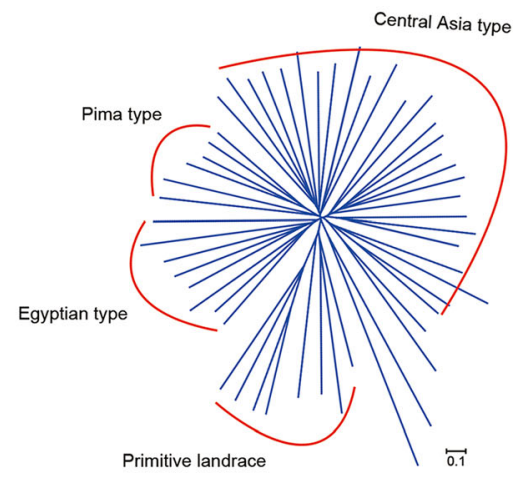

Fig. 1 Phylogenetic relationships of 147 cotton accessions. a A neighbor-joining tree was constructed using whole-genome SNP data. The cotton samples were divided into G. hirsutum races (orange), G. hirsutum cultivars (green), G. barbadense cultivars (dark blue) and outgroup species (light blue). $\mathbf{b}$ Population structure of cotton accessions determined using STRUCTURE. The accessions were divided into three groups when $\mathrm{K}=3$.

c Principal component analysis of all cotton accessions using whole-genome SNP data. d Phylogenetic relationships between G. hirsutum cultivars and races. e Phylogenetic relationships between G. barbadense landraces and cultivars. The scale bar indicates the simple matching distance

frequency distributions of 44,250 nearly fixed cSNPs were highly diverged between G. hirsutum and G. barbadense. The number of nearly fixed cSNPs detected between 33 race accessions and 52 cultivars in G. hirsutum was 1179 (Additional file 1: Figure S6). The sequence divergence at the evolution level among accessions was further evaluated using the ratio of nonsynonymous $(K a)$ SNPs against synonymous $(K s)$ SNPs. The average $K a / K s$ ratio was 0.49 for all common cSNPs. However, for 561 genes with nucleotide-binding site leucine-rich repeat domains, the ratio (0.73) was relatively higher, suggesting these genes are evolving more rapidly in response to co-evolving pathogens. The $\mathrm{Ka} / \mathrm{Ks}$ ratios for the nearly fixed cSNPs were 0.57 between G. hirsutum and G. barbadense, and 0.91 
between races and modern cultivars of G. hirsutum, indicating the existence of higher selection pressure during upland cotton domestication from wild to dooryard types and then field production.

We also identified 5784 protein-coding genes with premature stop codons or frameshifts resulting from 6661 SNPs and 2047 indels. A frameshift mutation occurred in a total of 1447 protein-coding genes resulting from 2047 indels (Additional file 9: Table S7). Of these, we found a flowering-related gene, Gh_D02G1411, homologous to ABA OVERLY SENSITIVE 4 (AtABO4, AT1G08260) in Arabidopsis. The abo4-1 plants were early flowering with lower expression of FLOWER LOCUS C and higher expression of FLOWER LOCUS $\mathrm{T}$ and changed histone modifications in these two loci [25]. Another interesting indel-containing gene encoding a cell wall-loosening protein, expansin A8 (EXPA8), played an important role in determining the rate and temporal period of fiber elongation and further fiber quality improvement [26].

We examined the genetic diversity across the 26 chromosomes (Additional file 10: Table S8), and a strong signal of differentiation was observed at the whole genome level between G. hirsutum and G. barbadense accessions (Fig. 2 chromosomes A01 and D01 displayed as examples and Additional file 1: Figure S7). The fixation index values $\left(F_{\mathrm{ST}}\right)$ were 0.63 and 0.65 in the $\mathrm{A}$ and $\mathrm{D}$ subgenomes, respectively, which were slightly higher than that between indica and japonica rice subspecies $\left(F_{\mathrm{ST}}=0.55\right)$ [27] and much higher than that between G. hirsutum races and cultivars ( $F_{\mathrm{ST}}=0.10$ for both subgenomes).

Whole-genome analysis identified 109 selective sweeps that spanned $3.4 \%$ of the G. hirsutum cotton genome through the comparison of 33 accessions of seven races and 52 modern cultivars $\left(\pi_{\text {race }} / \pi_{\text {cultivar }}>25\right.$; Fig. 3; Additional file 11: Table S9). We investigated the genomic variation of G. barbadense at the 109 selective sweep regions identified in G. hirsutum. Compared with the sequence diversity at the whole genome level, the $G$. barbadense population did not show a significant change at the 109 selective sweeps $\left(\pi_{\text {sweep }}=0.00055\right.$ versus $\pi_{\text {ge- }}$ nome $=0.00056$ ), indicating different selection pressures on the G. hirsutum and G. barbadense genomes. These genomic data further support our previous view that the two species were domesticated independently $[1,8]$. The phenomenon is similar to the dual domestication processes in common beans, where two divergent populations of Phaseolus vulgaris were independently domesticated in Mesoamerica and South America [28], as well as in cultivated rice, where Oryza sativa and Oryza glaberrima were independently domesticated in Asia and Africa [29].

G. hirsutum and G. barbadense had similar levels of sequence diversity. The nucleotide diversity levels of the $\mathrm{A}$ and D subgenomes were 0.00075 and 0.00073, respectively, in G. hirsutum, and 0.00061 and 0.00051 , respectively, in G. barbadense. It is possible that these numbers have been underestimated because tetraploid cotton genomes have large proportions of repetitive sequences and paralogs [21] similar to those in other large-genome plants such as maize [30]. To provide an indication of the mapping resolution in genome-wide association studies, the decay rate of linkage disequilibrium (LD) was calculated. The average pairwise correlation coefficient $\left(r^{2}\right)$ dropped from 0.6 at $1 \mathrm{~kb}$ to 0.3 at $1000 \mathrm{~kb}$ in G. hirsutum. This slow LD decay might have resulted from inbreeding nature in cotton. Moreover, as expected, a slower LD decay rate was found in cultivars than in the wild species and primitive races (Additional file 1: Figure S8).

\section{Asymmetric introgression between G. hirsutum and G. barbadense}

In spite of the strong genetic divergence between G. hirsutum and G. barbadense, the interspecific hybrids of the two cultivated species are fertile and grow vigorously, and some $F_{1}$ hybrids are commercially produced [31]. Cotton breeders have worked diligently to introduce some desired alleles from one species to another in order to increase genetic diversity. To analyze introgression between tetraploid cottons, a recently developed " 3 population test" method [32, 33] was used for modeling. Among all possible scenarios, we found evidence of introgression events between G. hirsutum races and G. barbadense cultivars ( $\mathrm{f} 3=-0.1223, \mathrm{Z}$ score $=-253.4$; Additional file 12: Table S10). These introgression events were successfully traced using the population-scale genomic data generated in the present study (Additional file 1: Figure S9). On average, $0.2 \%$ genomic regions in 137 accessions (excluding the ten outgroup accessions) showed obvious introgression events (384 introgression events detected in at least two accessions) (Additional file 13: Table S11). Intriguingly, the introgression events were significantly biased towards the gene flow from $G$. hirsutum into $G$. barbadense than that from G. barbadense into G. hirsutum (265 versus 119, Fisher's exact test, $P=8.04 \mathrm{E}-08$; Fig. 2; Additional file 14: Dataset 2). Moreover, more introgression events were found in the A subgenome (250) than in the D subgenome (134) (Fisher's exact test, $P=2.29$ E-05). A previous study described interspecies introgression in a limited population of $11 \mathrm{G}$. hirsutum and three G. barbadense [23]; however, the researchers used two diploid progenitor genomes $[34,35]$ instead of two published tetraploid genomes $[21,36]$ as the reference. Many structure variations have occurred after the formation of tetraploid cotton compared to two corresponding progenitors. From our previous colinearity analysis, the overall gene order and colinearity were largely conserved between our A and D subgenomes [21] and the D progenitor genome [34], but this colinearity was not 

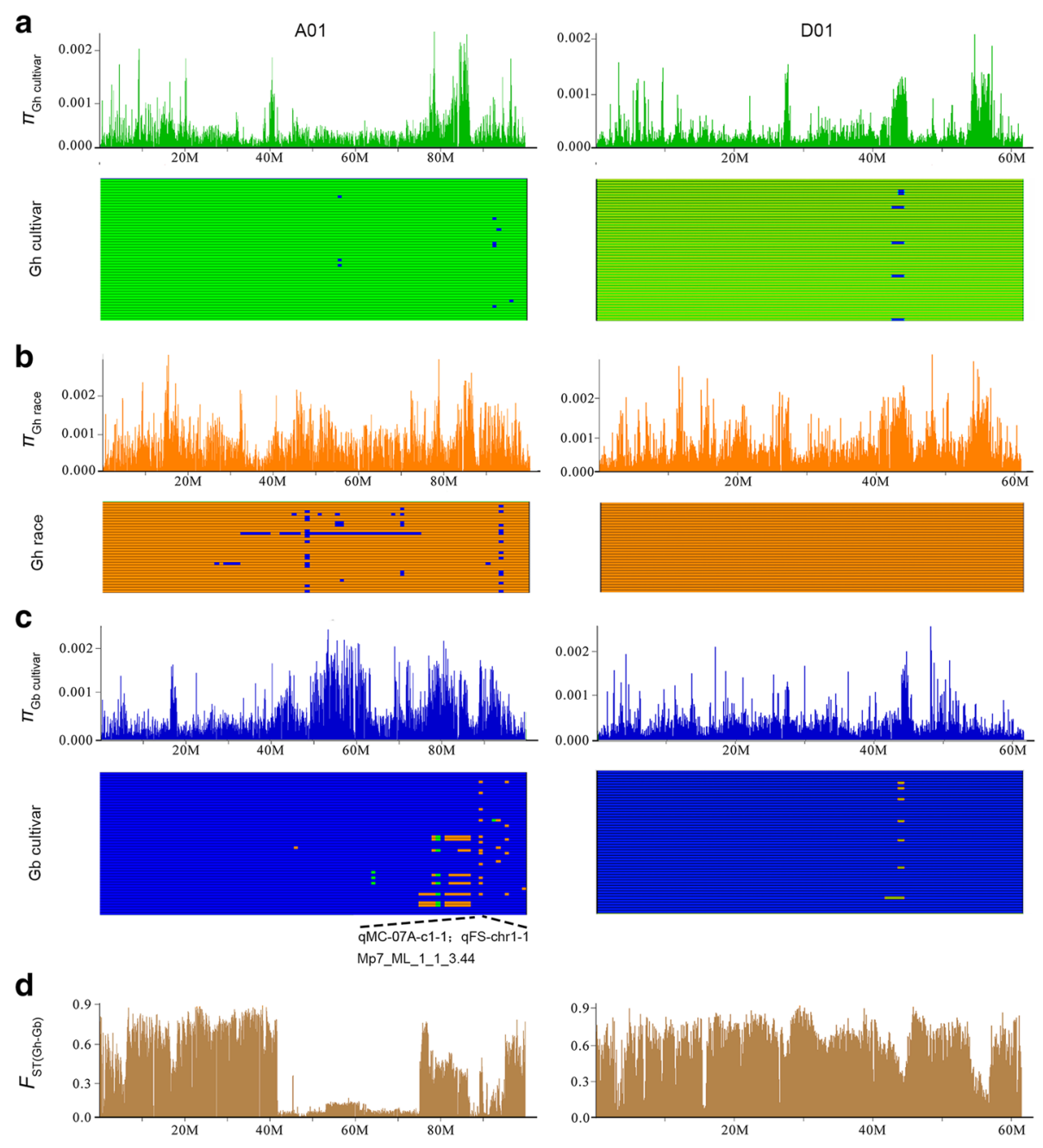

Fig. 2 Characterization of the genetic diversity and introgression on chromosomes A01 and D01 in cotton. The levels of genetic diversity in G. hirsutum cultivars ( $\pi_{G \text { h cultivar }}$ ( $(\mathbf{a})$ and races $\left(\pi_{G h}\right.$ race $)(\mathbf{b})$, the level of genetic diversity in $G$. barbadense $\left(\pi_{G b}\right.$ cultivar $)$ (c), and the level of genetic differentiation between G. hirsutum and G. barbadense (d). For introgression analysis, the genetic backgrounds of G. hirsutum cultivars, G. hirsutum races, and G. barbadense cultivars are illustrated in green (a), orange (b) and blue (c), respectively

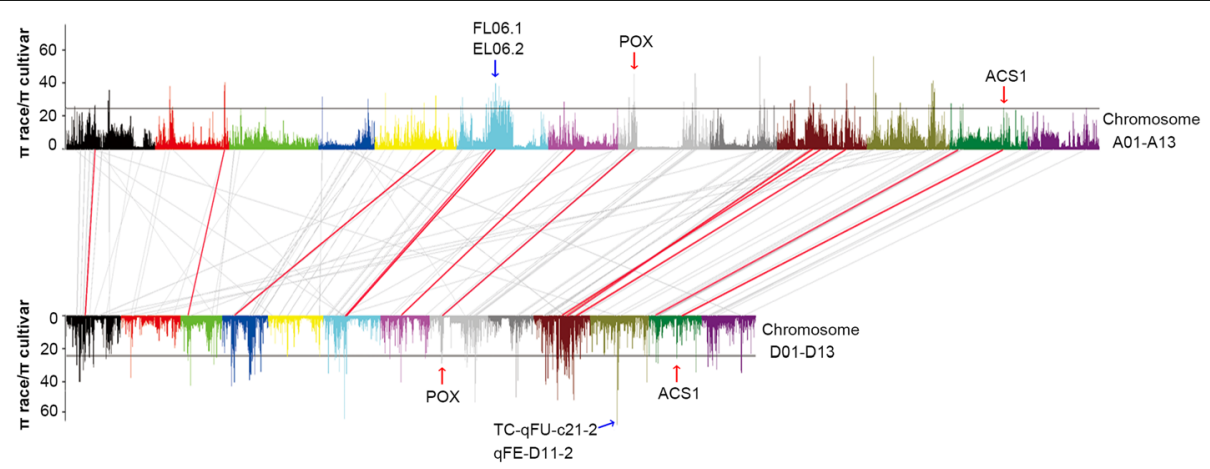

Fig. 3 Identification and comparative analysis of the selective sweeps in G. hirsutum. The values of $\pi_{\text {race }} / \pi_{\text {cultivar }}$ were plotted against the position on each of the 26 chromosomes. The relationships between each selective sweep and its corresponding homologous region in the allotetraploid genome are indicated by grey lines. The 12 selective sweep pairs with high or modest selection signals in homoeologous regions are indicated by red lines. The blue arrow indicates the fiber quality related QTLs around the strongest selection signal locus in D11 and the longest selection region in A06. The red arrow indicates the POX and ACS1 genes in the A08/D08 and A12/D12 homoeologous regions 
obvious between our A and D subgenomes and the A progenitor genome [35], partly due to numerous examples of mis-assemblies in the A progenitor genome, as we reported before [21], and partly because G. arboreum is an important cultivated diploid species and may have undergone some of its own chromosomal rearrangements during its evolution and improvement. Additionally, a larger population in the present study will be helpful to identify the introgression event more comprehensively compared to a previous study that used limited samples [23].

Across the allotetraploid cotton genomes, we found 11 regions of extensive introgressions, with the greatest density in chromosome A1 (Fig. 2; Additional file 14: Dataset 2; Additional file 15: Table S12). Analysis of QTLs has provided genetic evidence that these regions were associated with fiber quality traits (Additional file 16: Table S13). We observed 169 introgression events from six primitive races of G. hirsutum into Sea Island cottons of the G. barbadense species, such as Coastland R4-4, Seabrook, and West of Andes, instead of Tanquis, whose fiber was medium staple (23.8 to $27.0 \mathrm{~mm}$ in length) and was coarse. This fiber performance of the landraces such as Tanquis is typified by current cottons of Peru, where the ancestral G. barbadense originated [9]. Genomic evidence from the present study reveals subsequent introgressions from the local wild G. hirsutum or races into G. barbadense during its movement northward through inland Mesoamerica, from the Yucatan peninsula to the Caribbean Islands, where Sea Island cotton originally formed and was then introduced to the coastal states of the southeastern United States (Additional file 1: Figure S10). No introgression evens occurred from richmondi to Sea Island cottons, probably because of restricted geographical positions along the Pacific side of the Isthmus of Tehuantepec or limited collected accessions.

Among these 169 introgression events from G. hirsutum races into G. barbadense accessions, four events observed in Giza36, Giza80, Pima S-1, and Pima S-2 were detected in the same introgression region, the ChrA10.57.block (Additional file 14: Dataset 2). This block overlaps a QTL for fiber length (qFL-A10-2) [37]. In this block, we annotated 11 genes, of which five were potentially related to seed and fiber development, mainly involved in auxin transport (auxin efflux carrier gene) [38], transcription factors (WD40 repeat-like superfamily genes) [39, 40], and carbohydrate metabolism (o-fucosyltransferase gene, sucrose phosphate synthase gene, and beta galactosidase) [41] (Additional file 17: Table S14). In the ChrA11.88.block, which is also an introgression region from G. hirsutum races into four central Asia type G. barbadense accessions (CCCP1243, XH 3, XH 11, and XH 29), at least nine of 27 genes are potentially related to disease resistance, including two TIR-NBS-LRR genes [42], five pectin methylesterase inhibitor genes [43], and two dirigent-like protein genes [44] (Additional file 14: Dataset 2; Additional file 17: Table S14). We found 1061 genes in 169 introgression events from $G$. hirsutum races to $G$. barbadense and 665 genes in 96 events from G. hirsutum cultivars to G. barbadense. Interestingly, the genes in the former were enriched in developmental processes, such as reproduction, epithelial cell development, and cell proliferation, possibly allowing the allopolyploid to survive and even thrive considering its wide adaption. In contrast, the latter genes were enriched in cellular homeostasis, fatty acid oxidation, and lipid catabolic processes (Additional file 18: Table S15). In the 119 introgression events from $G$. barbadense to G. hirsutum, we further found 587 genes enriched in lipid metabolic and carbohydrate metabolic processes (Additional file 18: Table S15). These results support the idea that such introgressions confer beneficial traits such as fiber quality and photoperiod neutrality and are responsible for the creation of the Sea Island cotton germplasm, as reported previously $[5,9,12,20,31]$.

In spite of a low introgression rate, some G. barbadense segments were found to be introgressed into $G$. hirsutum races (Additional file 14: Dataset 2). These interspecific gene flows might have occurred during the northward movement of G. barbadense (Additional file 1: Figure S10).

Modern Egyptian-type ELS cultivars showed genomic signatures of G. hirsutum race introgressions in chromosome A1 (81-84 Mb, 88-89 Mb), A10 (21-22 Mb, 56$57 \mathrm{Mb})$, and $\mathrm{D} 11(10-11 \mathrm{Mb})$; the American-Pima type in $\mathrm{A} 1$ (77-78 Mb, 84-89 Mb) and A10 (56-57 Mb); and the Central Asia type in D1 (42-44 Mb), D9 (3-4 Mb, 5-6 Mb, 49-50 Mb), D10 (6-7 Mb, 57-62 Mb), and D11 (11-16 Mb, 63-64 Mb) (Additional file 13: Table S11; Additional file 14: Dataset 2), suggesting a distinct improvement in the Central Asian type ELS cultivars. Some introgression events, such as those in chromosome A1, were previously reported using restriction fragment length polymorphism markers [20], in which the G. hirsutum allele was found in 48 (94\%) of the 51 G. barbadense collections, including Egyptian and Pima cottons. Furthermore, modern breeding has enhanced gene flow and post-domestication introgressions through deliberate hybridization between these two species. For example, targeted introgressions from G. barbadense cultivars have been used to develop Acala cultivars, which improved upland cotton's fiber quality and Verticillium resistance [45].

\section{Signatures of selection and adaptive trait associations in G. hirsutum}

The genetic diversity in modern cultivars was found to be low $\left(\pi_{\text {cultivar }}=0.00074\right)$-only $34.2 \%$ (32.4 and $35.0 \%$ for the $\mathrm{A}$ and $\mathrm{D}$ subgenomes, respectively) of that in races 
$\left(\pi_{\text {race }}=0.00216\right)$-indicating a strong genetic bottleneck during upland cotton domestication. This diversity level is close to that in japonica rice (33\%) [27] and much lower than that in maize (83\%) [46] and indica rice (75\%) [27].

Phylogenetic analysis of the 109 selective sweeps revealed a strong selection pressure in nearly all cultivars of G. hirsutum. The average selection signal $\left(\pi_{\text {race }} / \pi_{\text {culti- }}\right.$ $\left.v_{v a r}=32.8\right)$ in the A subgenome was close to that in the D subgenome $\left(\pi_{\text {race }} / \pi_{\text {cultivar }}=35.0\right)$, but the sweep regions between the $\mathrm{A}$ and $\mathrm{D}$ subgenomes were largely different. These selective sweeps domesticated for fiber yield and fiber qualities provide a resource for molecular breeding of $G$. barbadense in the future.

Interestingly, 12 homoeologous pairs of selective sweeps with high or modest selection signals $\left(\pi_{\text {race }} / \pi_{\text {cultivar }}\right.$ ranging from 15.4 to 39.6) were detected between the A and D subgenomes (Fig. 3), probably due to selection of a common set of domestication genes. For example, peroxidase genes (POX, Gh_A08G0711/Gh_D08G0829) and ACC synthase genes (ACS1, Gh_A12G0969/Gh_D12G1017) participating in ethylene biosynthesis were co-selected within the overlapped regions of the selective sweeps of the A08/D08 and A12/D12 homoeologous pairs, and these genes play key roles in fiber elongation $[47,48]$.

To investigate the contribution of selective sweeps in the domestication for fiber yield and fiber qualities in $G$. hirsutum, the overlap between selective sweeps and QTLs of various agronomic traits was further examined. A total of 211 fiber quality- and lint yield-related QTLs were around 67 selective sweeps (Additional file 19: Table S16). The locus associated with the strongest selection signal $\left(\pi_{\text {race }} / \pi_{\text {cultivar }}=100.0\right)$ was located on chromosome D11 and overlapped with several QTLs controlling fiber length (Fig. 3; Additional file 19: Table S16). Another strong selective sweep was located on chromosome A6, covering a very long genomic interval $(21.6 \mathrm{Mb})$ that overlapped QTLs for fiber length and lint percentage (Additional file 19: Table S16). Fiber length and lint yield have greatly increased during domestication from wild type, primitive races, and advanced types to modern cultivars.

The examination of gene expression in selective sweeps responsible for various agronomic trait QTLs indicated some casual genes may be related to this domestication. Of the 1058 genes identified in all 109 selective sweeps, 723 were expressed in fiber development stages. Additionally, 236 of these 723 genes had significantly higher expression levels during fiber development in domesticated cotton (TM-1) than those in two wild relatives (TX665, G. hirsutum var. palmeri and TX2094, G. hirsutum var. yucatanense) (Additional file 20: Table S17).

Using RNA-seq data from multiple tissues, we found that the proportions of genes that were expressed during fiber development and seed germination were higher in the selective sweeps than in the whole genome (Additional file 1: Figure S11). Within the selective sweeps, 76 fiber- and 115 seed germination-related genes (Additional file 21: Table S18; Additional file 22: Table S19; Additional file 23: Table S20) were identified based on their expression profiles. Ten of these 76 genes were expressed at significantly higher level in TM-1 than in palmeri and yucatanense races (Fig. 4). For instance, a cytokinin oxidase gene (CKX6, Gh_D04G0688) was associated with increased fiber and seed yield [49]; a fatty acid desaturase (FAD3, Gh_07G0946) was required for the specific membrane structure of fiber cells and genes encoding very long chain fatty acid (VLCFA) synthase for fiber cell elongation $[47,48]$. These results suggest potential roles in the improved fiber qualities of domesticated cotton.

Of the 115 seed germination-related genes, Gene Ontology analysis showed an enrichment for genes involved in biological processes related to histone methylation and ethylene signaling pathways, which are required for the positive regulation of seed dormancy [50] (Additional file 23: Table S20). For instance, the gene encoding an AP2/ethylene response factor (ERF1, Gh_D10G1537) was found in the selective sweeps. The loss-of-function ap2 mutant showed increased seed mass relative to the wild type in Arabidopsis [51]. Overexpression of OsERF1 in Arabidopsis up-regulated the expression of two known ethylene-responsive genes, leading to short hypocotyls/ roots and the production of fewer seeds or no siliques at all [52]. Another gene, Gh_A10G0771, was homologous to a RING E3 ubiquitin ligase in Arabidopsis, which regulated the stability of the cyclin-dependent kinase inhibitor KRP1 and further negatively regulated the cell number and seed size [53, 54]. KRP1 is the target of the ubiquitinproteasome pathway recently found to play an important part in plant seed size determination [55, 56]. However, the molecular mechanisms of antagonistic function in the complex regulation of seed dormancy are still unclear. The candidate genes identified in the selective sweeps are valuable for future functional analyses of seed dormancy reduction during domestication.

\section{Conclusions}

Resequencing and genome-wide analysis of diverse $G$. hirsutum and G. barbadense wild accessions and modern cultivars have provided a comprehensive genomewide assessment of a fiber crop and enabled us to better understand the evolution, diversity, and domestication of allotetraploid cottons. Strong genomic divergence between G. hirsutum and G. barbadense led to dual domestication events of these two cultivated species, while reciprocal, but asymmetric, introgression between them has greatly improved their productivity and fiber quality. Although both are commonly grown as fiber crops, they have been domesticated or improved toward different 


\section{a}

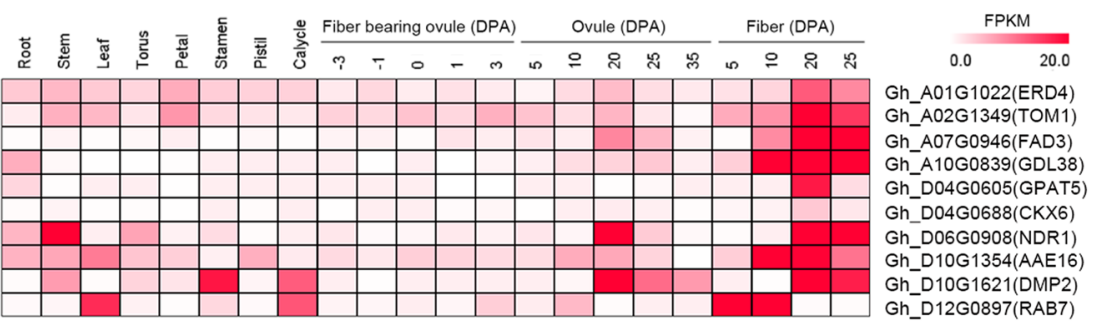

b

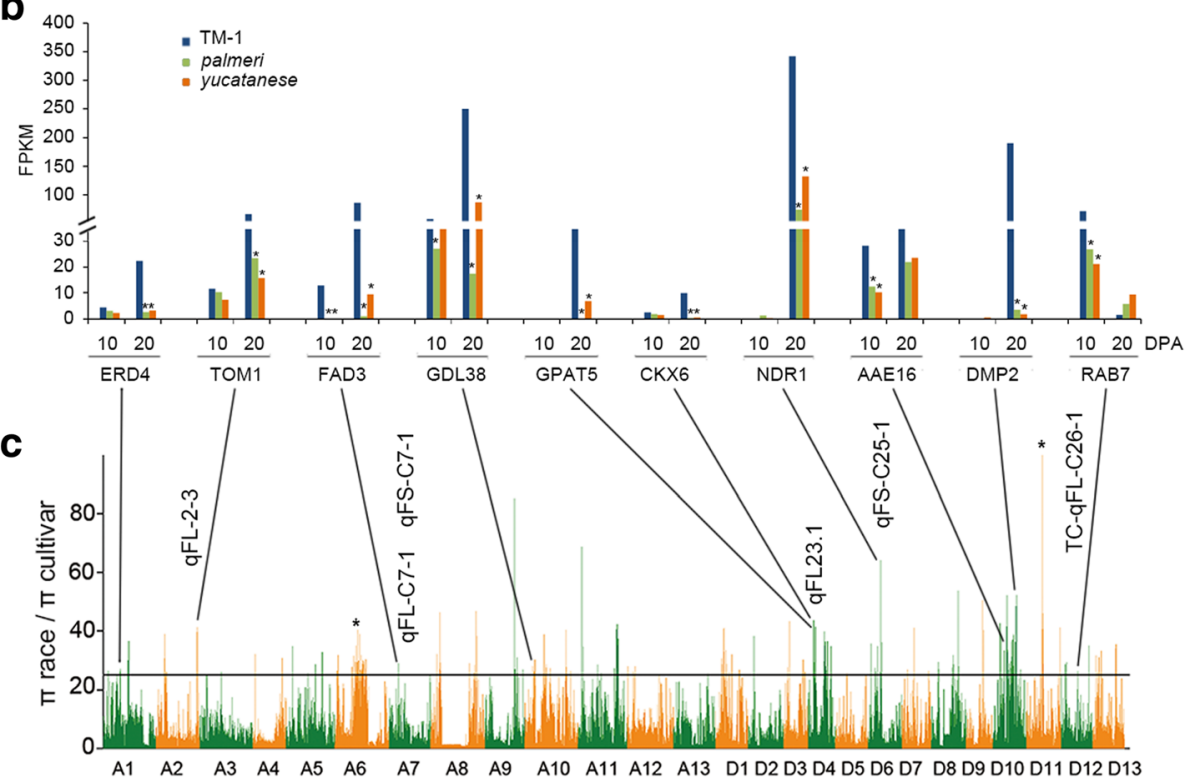

Fig. 4 Expression pattern of ten fiber-related genes related to cotton fiber quality. a Expression pattern of ten genes related to fiber quality in distinct tissues. b Expression level of these genes in domesticated cotton (TM-1) and two wild relatives (TX665, G. hirsutum var. palmeri; TX2094, G. hirsutum var. yucatanense). Fisher's exact test, ${ }^{*} P$ value $<0.05$ and fold change $>2$. c Identification of 109 selective sweeps through comparisons of races and cultivars in $G$. hirsutum. The values of $\pi_{\text {race }} / \pi_{\text {cultivar }}$ were plotted against the position on each of the 26 chromosomes. The horizontal line indicates the genome-wide threshold of selection signals $\left(\pi_{\text {race }} / \pi_{\text {cultivar }}>25\right)$. Asterisks indicate the strongest selection signal locus in D11 and the longest selective sweep in A06. Lines linking $\mathbf{b}$ and $\mathbf{c}$ indicate the gene locus in selection sweeps. The fiber quality-related QTLs around these gene loci are shown beside these lines

breeding goals: G. hirsutum for its high yield and wide adaptation, and G. barbadense for its superior fiber quality. This large amount of new genomic resources will substantially improve genetic mapping, gene identification, and molecular breeding in cotton. Specifically, under the guidance of sequence information, the favorable alleles that are associated with high yield potential and wide adaptation in G. hirsutum and with fiber quality in G. barbadense can be introgressed between the gene pools to further improve cotton production.

\section{Methods}

\section{Sampling}

In order to represent the rich genetic diversity and wide geographical distribution of cotton, we selected seven geographical races of G. hirsutum ("marie-galante', "punctatum", "richmond", "morrilli", "palmeri", "latifolium", and "yucatanense") [11], a variety of G. hirsutum cultivars, including four major types-Acala, Delta, Plains, and Eastern-from the USA, and other domesticated subtypes from Brazil, India, Africa, and China. Furthermore, G. barbadense cultivars, including American Pima, Egyptian, Peruvian Tanquis, and other subtypes from Russia and China, were also sampled. Although extant wild G. barbadense populations have been reported in Guayas and Los Rios in Ecuador and Tumbes in Peru [31, 57], the search for truly wild accessions is complicated since the wild-todomesticated continuum in G. barbadense does not have obvious categorical distinctions. Another three wild allotetraploid species, G. darwinii, native to the Galapagos Islands, G. tomentosum from the Hawaiian Islands, and G. mustelinum, an uncommon species restricted to a relatively small region of northeast Brazil, as well as Thespesia populneoides (Roxb.) Kostelas, a species in the mallow family (Malvaceae) closely related to the cotton genus (Gossypium), were chosen to form an outgroup. Detailed 
information on the 147 cotton accessions is listed in Additional file 2: Table S1.

\section{Library construction and sequencing}

For each cotton accession, young leaf tissues from a single plant were collected for genomic DNA extraction using a standard cetyl trimethylammonium bromide (CTAB) protocol [58]. Paired-end sequencing libraries with insert sizes ranging from 300 to 500 bp were constructed according to the manufacturer's instructions (Illumina, San Diego, CA, USA). All libraries were sequenced on the Illumina HiSeq 2000 platform. A total of 1.8 terabases of genomic sequence data was also generated with an average $5 \times$ genome coverage for each cotton accession.

\section{Genotype calling and SNP identification}

All sequence reads were aligned against the reference genome sequence (G. hirsutum cv. TM-1) [21] using Smalt software (version 0.57, http://www.sanger.ac.uk/ resources/software/smalt/). The parameter for the read mapping was "smalt_x86_64 map -i 700 -j 50 -m 60". For the oriented 1.9-Gb genome sequence, $36.2 \%$ of reads were mapped to the A subgenome and $23.9 \%$ to the D subgenome for G. hirsutum. Additionally, $10.5 \%$ of the total reads were mapped to the A subgenome scaffold (326.3 Mb); $1.9 \%$ of the total reads were mapped to the D subgenome scaffold $(61.5 \mathrm{Mb}) ; 4.1 \%$ of the total reads were mapped to unclassified scaffold (124.6 Mb); and $23.4 \%$ of the total reads had no unique location in the mapping process. Only reads with a unique mapping position in the oriented reference genome and a mapping score higher than 60 were used. If reads had equal matching scores in the $\mathrm{A}$ and $\mathrm{D}$ subgenomes, the reads were excluded from the SNP calling procedure. The software package Ssaha pileup (http://www.sanger.ac.uk/resources/software/ssaha2/) was used to find candidate SNPs that required support from at least two sequence reads. Only the non-singleton SNPs, defined as those where more than two accessions demonstrate the presence of the alternative alleles, were retained. We then filtered the polymorphic sites with minor allele frequency (5\%) and missing rate (10\%). We randomly removed the polymorphic sites in the high homoeologous polymorphic sites until the remaining polymorphic sites were at least $10 \mathrm{bp}$ away from neighboring polymorphic sites and got the final polymorphic datasets. In the filtering process, we found that about $40 \%$ of non-singleton polymorphic sites had missing rates of less than $10 \%$. We required that the common SNPs had a minor allele frequency (MAF) greater than $5 \%$ and a missing data rate less than $10 \%$. We only analyzed the SNPs that were located in the 26 pseudomolecules of the TM-1 assembly, and the SNPs in the small scaffolds were removed.
The SNPs were annotated using the GFF files (the annotation file of all coding regions of each gene) of the TM-1 reference genome sequence. The software $\mathrm{KaKs}$ _Calculator was then used to compute the $\mathrm{Ka} / \mathrm{Ks}$ ratio.

\section{Indel identification and annotation}

Pindel software (version 0.20) [59] was used to identify the indels from the sequence reads. In order to identify the indels, we used the Smalt outputs and kept only three kinds of reads: (1) the paired-end sequence reads that had a unique match on one side and no match on the other side; (2) the paired-end reads where small indels were detected in the Smalt output file; and (3) the paired-end reads that had a unique match in the genome but a low alignment score. We converted the filtered reads into the Pindel input file format. Only the indels that had the support of more than three reads and were detected in at least two accessions were retained as candidate indels. The genomic position of each indel was checked against the GFF file to allow for cotton genome annotation. Genes with indels causing open reading frame changes were considered to have a mutation with a large effect.

\section{SNP validation}

We used two methods to validate the SNP calling. First, we used the assemblies of the TM-1 and XH21 genomes to identify the genotypes of TM-1 and XH21 at the SNP site, respectively. We compared the genotypes in the assembled sequences against those in the SNP datasets called from the resequencing data and calculated the SNP accuracy rate. Second, we further randomly selected 68 SNPs and carried out PCR-based sequencing in 11 randomly selected accessions (seven G. hirsutum and four $G$. barbadense accessions) with three replicates. We aligned all the PCR products against the TM-1 genome using BLAST (version 2.2.28), and the reads with mapping lengths $>90 \%$ and identity $>80 \%$ were used for SNP validation. Using the alignment results, we retrieved the genotypes in 11 accessions for each SNP site. Only the genotypes consistent across three replicates were used to calculate the accuracy (Additional file 24: Table S21; Additional file 25: Table S22).

\section{Population structure analysis}

Using the Ssaha pileup package, we generated an SNP matrix for 147 cotton accessions and calculated the simple matching coefficient of whole-genome SNPs as the genetic distance. We used Phylip software (version 3.69) [60] to generate the neighbor-joining tree. Dendroscope [61] was used to display the phylogenetic tree. The missing data in the cotton SNP genotype dataset were imputed using Beagle (version 3.3.2) [62]. We converted 
the raw genotype matrix to the Beagle unphased format and imputed using the parameter "-unphased". We performed population structure analysis using STRUCTURE [63] and principal component analysis was performed using EIGENSTRAT software [64].

\section{Population genetics analysis}

According to the phylogenetic tree, the 147 cotton accessions could be divided into four major groups: $G$. hirsutum races, G. hirsutum cultivars, G. barbadense cultivars, and the outgroup. For each group, we calculated the level of genetic diversity $(\pi)$ in each $100-\mathrm{kb}$ interval across the cotton genome. The level of population differentiation, $F_{\mathrm{ST}}$, was calculated in $100-\mathrm{kb}$ intervals. During the identification of selective sweeps in $G$. hirsutum cultivars, we measured the level of genetic diversity in races and in cultivars. We used the ratio $\pi_{\text {race }} / \pi_{\text {cultivar }}$ to evaluate selection signals. Genomic regions with diversity values lower than 0.001 in both races and cultivars were removed from the ratio calculation. Permutation tests were performed to estimate the false positive rate of the selection sweeps in order to validate the accuracy of the diversity ratio, with a ratio of 25 used as the threshold [65]. LD was calculated using Haploview software, with the default settings [66]. To model the mixture between different populations, the software TreeMix was used to perform a "3-population test" $[32,33]$. In the test, the $\mathrm{f} 3$ statistic is the normalized product of the frequency difference. If there is no mixture, the expected value of the $\mathrm{f} 3$ statistic is positive.

\section{Introgression between G. hirsutum and G. barbadense}

We screened 26 cotton chromosomes to detect the genomic regions of genetic introgression. A recently developed "3-population test" method [32, 33] was used for modeling the introgression between tetraploid cottons. Moreover, we divided the whole cotton genome into 1$\mathrm{Mb}$ blocks and calculated the genetic distances through simple matching coefficients between the 147 cotton accessions. Within the introgression regions, we detected a total of 384 introgression events that were observed in at least two accessions. Phylip software (version 3.69) was used to generate the $\mathrm{NJ}$ tree, and the R software package "ape" was used to display the tree file. We checked each phylogenetic tree and searched for $G$. hirsutum accessions located within the G. barbadense clade or G. barbadense accessions located within the G. hirsutum clade.

\section{Calculation of gene expression level}

RNA-Seq data from distinct tissues have been reported in previous TM-1 genome sequencing research [21]. The raw transcriptome data of two wild cotton relatives (TX665, G. hirsutum var. palmeri; TX2094, G. hirsutum var. yucatanense) were downloaded from the Sequence Read Archive (http://www.ncbi.nlm.nih.gov/sra/SRX202873) [67]. We calculated the expression of each gene using fragments per kilobase of exon model per million mapped reads (FPKM) with Cufflinks (version 2.1.1) [68]. Gene expression in different tissues is listed in Additional file 21: Table S18. Genes that had different transcript levels among these 14 tissues were defined at $P<0.05$ using a Student's $t$-test. If the gene expression in one tissue did not represent a 95\% confidence level of the $\mathrm{T}$ distribution of the other 13 tissues, it was identified as a special tissue-related gene.

\section{Additional files}

Additional file 1: Supplementary Figures S1-S11. (DOCX 4378 kb)

Additional file 2: Table S1. Summary of the 147 cotton samples and their sequencing data. (XLSX $28 \mathrm{~kb}$ )

Additional file 3: Table S2. The 68 SNPS validated in 11 randomly selected accessions by PCR methods. (XLSX $20 \mathrm{~kb}$ )

Additional file 4: Table S3. SNP accuracy verified using PCR-based sequencing. (XLSX $10 \mathrm{~kb}$ )

Additional file 5: Table S4. Summary of detected indels based on the genome resequencing data. (XLSX 6692 kb)

Additional file 6: Table S5. Summary of detected SVs based on the genome resequencing data. (XLSX $837 \mathrm{~kb}$ )

Additional file :7 Table S6. Distribution of SNPs and indels in 26 chromosomes of TM-1. (XLSX $11 \mathrm{~kb}$ )

Additional file 8: Dataset 1. A phylogenetic tree with full accession names. (PDF $180 \mathrm{~kb}$ )

Additional file 9: Table S7. Summary information of 1447 indelcontaining genes. (XLSX 532 kb)

Additional file 10: Table S8. Levels of genetic differentiation in different chromosomes. (XLSX $11 \mathrm{~kb})$

Additional file 11: Table S9. Genome-wide detection of selective sweep regions in upland cotton domestication. (XLSX $25 \mathrm{~kb}$ )

Additional file 12: Table S10. The results of the 3-population test. (XLSX $11 \mathrm{~kb}$ )

Additional file 13: Table S11. Genomic regions with introgressions between $\mathrm{G}$. barbadense and G. hirsutum. (XLSX $25 \mathrm{~kb}$ )

Additional file 14: Dataset 2. Whole-genome analysis of genetic introgressions in allotetraploid cotton. (PDF $111 \mathrm{~kb}$ )

Additional file 15: Table S12. Genomic regions with extensive introgressions between G. barbadense and G. hirsutum. (XLSX $12 \mathrm{~kb}$ )

Additional file 16: Table S13. Cotton QTLs that overlapped with the introgression regions. (XLS $81 \mathrm{~kb}$ )

Additional file 17: Table S14. Candidate genes in two introgression regions from $G$. hirsutum race to $G$. barbadense. (XLSX $10 \mathrm{~kb}$ )

Additional file 18: Table S15. Functional enrichment of genes involved in introgression events between G. hirsutum and G. barbadense. (XLSX $11 \mathrm{~kb}$ )

Additional file 19: Table S16. List of fiber quality- and lint yield-related QTLs overlapping selected sweeps. (XLSX 34 kb)

Additional file 20: Table S17. Differently expressed selective sweep related genes between domesticated cotton and wild relatives. (XLSX $26 \mathrm{~kb}$ )

Additional file 21: Table S18. Gene expression level of 1058 genes identified in 109 selection sweeps. (XLSX 345 kb) 
Additional file 22: Table S19. List of genes related to cotton fiber development that overlapped with the selective sweeps in G. hirsutum (XLSX $14 \mathrm{~kb}$ )

Additional file 23: Table S20. List of genes related to cotton seed germination that overlapped with the selective sweeps in G. hirsutum. (XLSX $17 \mathrm{~kb})$

Additional file 24: Table S21. Primers for SNP accuracy validation. (XLSX $12 \mathrm{~kb}$ )

Additional file 25: Table S22. The position of 68 SNPs located in the 24 regions amplified by PCR. (XLSX $12 \mathrm{~kb})$

\section{Acknowledgements}

We thank Dr. J Udall for his insightful comments. We thank the National Medium-term Gene Bank of Cotton in China for providing some of the cotton germplasm resource seeds and permitting the harvest of the leaves of seven $G$. hirsutum races to isolate the DNA used in the present study.

\section{Funding}

This work was financially supported in part by grants from NSFC (31330058), National Key R \& D Program for Crop Breeding (2016YFD0100306), the Priority Academic Program Development of Jiangsu Higher Education Institutions, the 111 project (B08025), and the JCIC-MCP project.

\section{Availability of data and materials}

Sequences have been deposited at the NCBI under the accessions PRJNA248163, SRP047301, and SRP044705.

\section{Authors' contributions}

TZ conceptualized the research program. TZ, XH, XC, WG, and BH designed experiments and coordinated the project. $\mathrm{TZ}, \mathrm{BL}$, and $\mathrm{XD}$ collected the 147 cotton samples. CL, YK, MP, LC, QW, GM, and XL extracted high quality DNA from all 147 samples. LF, YH, YW, and CL constructed DNA sequencing libraries and performed the genome sequencing. $\mathrm{HG}, \mathrm{LF}, \mathrm{XH}, \mathrm{CL}, \mathrm{TH}, \mathrm{JC}$, and QuW performed the genotyping and other bioinformatics analyses. LF, CL, $\mathrm{YH}, \mathrm{SW}, \mathrm{QW}, \mathrm{BL}, \mathrm{HW}, \mathrm{SC}, \mathrm{DX}, \mathrm{CC}$, and XZ were involved in the data analysis. $\mathrm{TZ}, \mathrm{XH}, \mathrm{XC}, \mathrm{WG}$, and $\mathrm{BH}$ supervised data generation and analysis. $\mathrm{TZ}, \mathrm{XH}, \mathrm{WG}$, $L F, C L, Y H, H G, B H, Z J C$, and DF analyzed all of the data and wrote the manuscript. All authors discussed results and commented on the manuscript. All authors read and approved the final manuscript.

\section{Competing interests}

The authors declare that they have no competing interests.

\section{Ethics approval and consent to participate}

Not applicable.

\footnotetext{
Author details

'State Key Laboratory of Crop Genetics and Germplasm Enhancement, Cotton Hybrid R \& D Engineering Center (the Ministry of Education), Nanjing Agricultural University, Nanjing 210095, China. ${ }^{2}$ National Center for Gene Research, Institute of Plant Physiology and Ecology, Shanghai Institutes for Biological Sciences, Chinese Academy of Sciences, Shanghai 200233, China. ${ }^{3}$ Cotton Fiber Bioscience Research Unit, USDA-ARS-SRRC, New Orleans, LA 70124, USA. ${ }^{4}$ State Key Laboratory of Cotton Biology, Institute of Cotton Research of the Chinese Academy of Agricultural Sciences, Anyang, China. ${ }^{5}$ Cotton Research Institute, Xinjiang Academy of Agriculture and Reclamation Sciences, Xinjiang 832000, China. 'Department of Molecular Biosciences, Center for Computational Biology and Bioinformatics, and Institute for Cellular and Molecular Biology, the University of Texas at Austin, Austin, TX 78712, USA. ${ }^{7}$ State Key Laboratory of Plant Molecular Genetics, Institute of Plant Physiology and Ecology, Shanghai Institutes for Biological Sciences, Chinese Academy of Sciences, Shanghai 200032, China. ${ }^{8}$ Agronomy Department, College of Agriculture and Biotechnology, Zhejiang University, Zhejiang 310029, China. ${ }^{9}$ College of life and environmental sciences, Shanghai Normal University, Shanghai 200234, China.
}

Received: 17 December 2016 Accepted: 6 February 2017

Published online: 20 February 2017

\section{References}

1. Hutchinson JB. The application of genetics to cotton improvement. Cambridge: Cambridge University Press; 1959

2. Kohel RJ, Lewis CF. Cotton. Madison: American Society of Agronomy; 1984.

3. Wendel JF. New World tetraploid cottons contain Old-World cytoplasm. Proc Natl Acad Sci U S A. 1989:86:4132-6.

4. Fryxell PA. Natural history of the cotton tribe. College Station, TX: Texas A\&M University Press; 1979; Suppl 74:4-7

5. Stephens SG. Some observations on photoperiodism and the development of annual forms of domesticated cottons. Econ Bot. 1976;30:409.

6. Stephens SG. The effects of domestication on certain seed and fiber properties of perennial forms of cotton. Gossypium hirsutum L Am Nat. 1965;99:17.

7. McGarry RC, Prewitt SF, Culpepper S, Eshed Y, Lifschitz E, Ayre BG. Monopodial and sympodial branching architecture in cotton is differentially regulated by the Gossypium hirsutum SINGLE FLOWER TRUSS and SELFPRUNING orthologs. New Phytol. 2016. doi:10.1111/nph.14037.

8. Wendel JF, Brubaker CL, Seelanan T. The origin and evolution of Gossypium. In: Stewart JM, Oosterhuis DM, Heitholt JJ, Mauney JR, editors. Physiology of cotton. Netherlands: Springer; 2010. p. 1-18.

9. Percy RG, Wendel JF. Allozyme evidence for the origin and diversification of Gossypium barbadense L. Theor Appl Genet. 1990;79:529-42.

10. Westengen OT, Huaman Z, Heun M. Genetic diversity and geographic pattern in early South American cotton domestication. Theor Appl Genet. 2005:110:392-402.

11. Hutchinson JB. Intra-specific differentiation in Gossypium hirsutum. Heredity. 1951;5:24.

12. Doebley JF, Gaut BS, Smith BD. The molecular genetics of crop domestication. Cell. 2006:127:1309-21.

13. Applequist WL, Cronn R, Wendel JF. Comparative development of fiber in wild and cultivated cotton. Evol Dev. 2001;3:3-17.

14. May OL, Bowman DT, Calhoun DS. Genetic diversity of US upland cotton cultivars released between 1980 and 1990. Crop Sci. 1995;35:1570-4.

15. Van Esbroeck GA, Bowman DT, May OL, Calhoun DS. Genetic similarity indices for ancestral cotton cultivars and their impact on genetic diversity estimates of modern cultivars. Crop Sci. 1999:39:323-8.

16. Wendel JF, Brubaker CL, Percival AE. Genetic diversity in Gossypium hirsutum and the origin of Upland cotton. Am J Bot. 1992;79:1291-310.

17. Brubaker CL, Wendel JF. Reevaluating the origin of domesticated cotton (Gossypium hirsutum, Malvaceae) using nuclear restriction fragment length polymorphisms (RFLP). Am J Bot. 1994;81:1309-26.

18. Lacape JM, Dessauw D, Rajab M, Noyer JL, Hau B. Microsatellite diversity in tetraploid Gossypium germplasm: assembling a highly informative genotyping set of cotton SSRs. Mol Breed. 2007;19:45-58.

19. Tyagi P, Gore MA, Bowman DT, Campbell BT, Udall JA, Kuraparthy V. Genetic diversity and population structure in the US Upland cotton (Gossypium hirsutum L.). Theor Appl Genet. 2014:127:283-95.

20. Wang G, Dong J, Paterson AH. The distribution of Gossypium hirsutum chromatin in Gossypium barbadense germ plasm: molecular analysis of introgressive plant breeding. Theor Appl Genet. 1995;91:1153-61.

21. Zhang T, Hu Y, Jiang W, Fang L, Guan X, Chen J, et al. Sequencing of allotetraploid cotton (Gossypium hirsutum L. acc. TM-1) provides a resource for fiber improvement. Nat Biotechnol. 2015:33:531-7.

22. Liu X, Zhao B, Zheng H, Hu Y, Lu G, Yang C, et al. Gossypium barbadense genome sequence provides insight into the evolution of extra-long staple fiber and specialized metabolites. Sci Rep. 2015;5:14139.

23. Page JT, Liechty ZS, Alexander RH, Clemons K, Hulsekemp AM, Ashrafi H, et al. DNA sequence evolution and rare homoeologous conversion in the tetraploid cotton. PLOS Genet. 2016;12:e1006012.

24. Coppens d'Eeckenbrugge G, Lacape JM. Distribution and differentiation of wild, feral, cultivated populations of perennial Upland cotton (Gossypium hirsutum L.) in Mesoamerica and the Caribbean. PLoS One. 2014;9:e107458

25. Yin H, Zhang $X$, Liu J, Wang $Y$, He J, Hong $X$, et al. Epigenetic regulation, somatic homologous recombination, and abscisic acid signaling are influenced by DNA polymerase \{epsilon\} mutation in Arabidopsis. Plant Cell. 2009;21:386-402. 
26. Li Y, Tu L, Pettolino F, Ji S, Hao J, Yuan D, et al. GbEXPATR, a species-specific expansin, enhances cotton fibre elongation through cell wall restructuring. Plant Biotechnol J. 2015;14:951-63.

27. Huang X, Wei X, Sang T, Zhao Q, Feng Q, Zhao Y, et al. Genome-wide association studies of 14 agronomic traits in rice landraces. Nat Genet. 2010;42:961-7.

28. Schmutz J, McClean PE, Mamidi S, Wu G, Cannon SB, Grimwood J, et al. A reference genome for common bean and genome-wide analysis of dual domestication. Nat Genet. 2014;46:707-13.

29. Wang M, Yu Y, Haberer G, Marri PR, Fan C, Goicoechea JL, et al. The genome sequence of African rice (Oryza glaberrima) and evidence for independent domestication. Nat Genet. 2014;46:982-8.

30. Chia JM, Song C, Bradbury PJ, Costich DE, De Leon N, Doebley J, et al. Maize HapMap2 identifies extant variation from a genome in flux. Nat Genet. 2012;44:803-7.

31. Percival AE, Kohel RJ. Distribution, collection, and evaluation of Gossypium. Adv Agron. 1990;44:225-56.

32. Myles S, Boyko AR, Owens CL, Brown PJ, Grassi F, Aradhya MK, et al. Genetic structure and domestication history of the grape. Proc Natl Acad Sci U S A. 2010;108:3530-5.

33. Reich D, Thangaraj K, Patterson N, Price AL, Singh L. Reconstructing Indian population history. Nature. 2009;461:489-94.

34. Paterson AH, Wendel JF, Gundlach $\mathrm{H}$, Guo H, Jenkins J, Jin D, et al. Repeated polyploidization of Gossypium genomes and the evolution of spinnable cotton fibres. Nature. 2012;492:423-7.

35. Li F, Fan G, Wang K, Sun F, Yuan Y, Song G, et al. Genome sequence of the cultivated cotton Gossypium arboreum. Nat Genet. 2014;46:567-72.

36. Li F, Fan G, Lu C, Xiao G, Zou C, Kohel RJ, et al. Genome sequence of cultivated Upland cotton (Gossypium hirsutum TM-1) provides insights into genome evolution. Nat Biotechnol. 2015;33:524-30.

37. Zhang T, Qian N, Zhu X, Chen H, Wang S, Mei H, et al. Variations and transmission of QTL alleles for yield and fiber qualities in Upland cotton cultivars developed in China. PLoS One. 2013;8:e57220.

38. Wang $M$, Zhao $P$, Cheng $H$, Han L, Wu X, Gao $P$, et al. The cotton transcription factor TCP14 functions in auxin-mediated epidermal cell differentiation and elongation. Plant Physiol. 2013;162:1669-80.

39. Walker AR, Davison PA, Bolognesi-Winfield AC, James CM, Srinivasan N, Blundell TL, et al. The TRANSPARENT TESTA GLABRA1 locus, which regulates trichome differentiation and anthocyanin biosynthesis in Arabidopsis, encodes a WD40 repeat protein. Plant Cell. 1999:11:1337-50.

40. Xiao Y, Wei L, Tao Q, Sun X. At1g65030, a WD40-Repeat protein gene, regulates seed mass and size in Arabidopsis. Plant Physiol. 2011;7:715-25.

41. Gou J, Wang L, Chen S, Hu W, Chen X. Gene expression and metabolite profiles of cotton fiber during cell elongation and secondary cell wall synthesis. Cell Res. 2007;17:422-34.

42. Mchale LK, Tan X, Koehl P, Michelmore RW. Plant NBS-LRR proteins: adaptable guards. Genome Biol. 2006;7:212.

43. Lionetti V, Cervone F, Bellincampi D. Methyl esterification of pectin plays a role during plant-pathogen interactions and affects plant resistance to diseases. Plant Physiol. 2012;169:1623-30.

44. Zhu L, Zhang X, Tu L, Zeng F, Nie Y, Guo X. Isolation and characterization of two novel dirigent-like genes highly induced in cotton (Gossypium barbadense and G. hirsutum) after infection by Verticillium dahliae. J Plant Pathol. 2007:89:41-5.

45. Lubbers EL, Chee PW. The worldwide gene pool of G. hirsutum and its improvement. In: Paterson AH, editor. Genetics and Genomics of Cotton. New York: Springer; 2009. p. 23-52.

46. Hufford MB, Xu X, Van Heerwaarden J, Pyhajarvi T, Chia J, Cartwright RA, et al. Comparative population genomics of maize domestication and improvement. Nat Genet. 2012;44:808-11.

47. Qin Y, Hu C, Pang Y, Kastaniotis AJ, Hiltunen JK, Zhu Y. Saturated very-longchain fatty acids promote cotton fiber and Arabidopsis cell elongation by activating ethylene biosynthesis. Plant Cell. 2007;19:3692-704.

48. Qin Y, Zhu Y. How cotton fibers elongate: a tale of linear cell-growth mode. Curr Opin Plant Biol. 2011;14:106-11.

49. Zhao J, Bai W, Zeng Q, Song S, Zhang M, Li X, et al. Moderately enhancing cytokinin level by down-regulation of GhCKX expression in cotton concurrently increases fiber and seed yield. Mol Breed. 2015;35:60.

50. Nonogaki $\mathrm{H}$. Seed dormancy and germination-emerging mechanisms and new hypotheses. Front Plant Sci. 2014;5:233.
51. Ohto MA, Fischer RL, Goldberg RB, Nakamura K, Harada JJ. Control of seed mass by APETALA2. Proc Natl Acad Sci U S A. 2005;102:3123-8.

52. Hu Y, Zhao L, Wang T. Overexpression of OsERF1, a novel rice ERF gene, up-regulates ethylene-responsive genes expression besides affects growth and development in Arabidopsis. Plant Physiol. 2008;165:1717-25.

53. Ren H, Santner A, Pozo JC, Murray JA, Estelle M. Degradation of the cyclindependent kinase inhibitor KRP1 is regulated by two different ubiquitin E3 ligases. Plant J. 2008:53:705-16.

54. Cheng Y, Cao L, Wang S, Li Y, Shi X, Liu H, et al. Downregulation of multiple CDK inhibitor ICK/KRP genes upregulates the E2F pathway and increases cell proliferation, and organ and seed sizes in Arabidopsis. Plant J. 2013;75:642-55

55. Du L, Li N, Chen L, Xu Y, Li Y, Zhang Y, et al. The ubiquitin receptor DA1 regulates seed and organ size by modulating the stability of the ubiquitinspecific protease UBP15/SOD2 in Arabidopsis. Plant Cell. 2014;26:665-77.

56. Li N, Li Y. Signaling pathways of seed size control in plants. Curr Opin Plant Biol. 2016;33:23-32.

57. Schwendiman J, Ano G, Percival AE. Cotton collecting in continental Ecuador and Galapagos Islands. Plant Genet Res News. 1986;64:33-7.

58. Paterson AH, Brubaker $\mathrm{C}$, Wendel JF. A rapid method for extraction of cotton (Gossypium spp.) genomic DNA suitable for RFLP or PCR analysis. Plant Mol Biol Rep. 1993:11:122-7.

59. Ye K, Schulz MH, Long Q, Apweiler R, Ning Z. Pindel: a pattern growth approach to detect break points of large deletions and medium sized insertions from paired-end short reads. Bioinformatics. 2009;25:2865-71.

60. Felsenstein J. PHYLIP: phylogeny inference package (version 3.2). Cladistics. 1989:5:164-6.

61. Huson DH, Richter DC, Rausch C, Dezulian T, Franz M. Dendroscope: An interactive view er for large phylogenetic trees. BMC Bioinformatics. 2007:8:460.

62. Browning BL, Browning SR. A unified approach to genotype imputation and haplotype phase inference for large data sets of trios and unrelated individuals. Am J Hum Genet. 2009:84:210-23.

63. Falush D, Stephens M, Pritchard JK. Inference of population structure using multilocus genotypes data: linked loci and correlated allele frequencies. Genetics. 2003;164:1567-87.

64. Price AL, Patterson NJ, Pienge RM, Weinblatt ME, Shadick NA, Reich D. Principal components analysis corrects for stratification in genome-wide association studies. Nat Genet. 2006;38:904-9.

65. Churchill GA, Doerge RW. Empirical threshold values for quantitative trait mapping. Genetics. 1994;138:963-71.

66. Barrett JC, Fry B, Maller J, Daly MJ. Haploview: analysis and visualization of LD and haplotype maps. Bioinformatics. 2005;21:263-5.

67. Yoo M, Wendel JF. Comparative evolutionary and developmental dynamics of the cotton (Gossypium hirsutum) fiber transcriptome. PLOS Genet. 2014; 10:1-19.

68. Trapnell C, Williams BA, Pertea G, Mortazavi A, Kwan G, Van Baren MJ, et al. Transcript assembly and quantification by RNA-Seq reveals unannotated transcripts and isoform switching during cell differentiation. Nat Biotechnol. 2010;28:511-5.

\section{Submit your next manuscript to BioMed Central and we will help you at every step:}

- We accept pre-submission inquiries

- Our selector tool helps you to find the most relevant journal

- We provide round the clock customer support

- Convenient online submission

- Thorough peer review

- Inclusion in PubMed and all major indexing services

- Maximum visibility for your research

Submit your manuscript at www.biomedcentral.com/submit 